\title{
Robust Patterns in Recurrent Sampling of Multiband Signals
}

\author{
Lihu Berman and Arie Feuer, Fellow, IEEE
}

\begin{abstract}
Periodic nonuniform sampling can be used to achieve sub-Nyquist sampling of bandlimited multiband signals. In this paper, we examine the question of selecting the sampling pattern in such a scheme, so that the reconstruction robustness-measured by the condition-number of the modulation matrix-is maximized. Contrary to previous work, where the sampling pattern was chosen from a discrete set, we let the sampling patterns vary continuously, but impose a structural constraint. Using this approach, we derive necessary and sufficient conditions on the spectral support of the signal for which perfect conditioning exist, namely, for which a sampling pattern can be found so that the resulting modulation matrix has a condition number equal to 1 . A simple test to check for these conditions is developed and the desired sampling patterns are found. An algorithm for choosing the sampling pattern when the aforementioned conditions are not satisfied is also introduced. Finally, we present some simulation results.
\end{abstract}

Index Terms-Condition number, multiband signals, nonuniform sampling.

\section{INTRODUCTION}

$\mathbf{S}$ AMPLING is a very important aspect of digital signal processing (DSP), being the means to connect the physical, analog world to the digital realm. The most common form is the uniform sampling of bandlimited signals [1], [2], where perfect reconstruction of a signal is possible from its uniform samples taken at a rate greater than $2 B$ (where $B$ is the signal bandwidth).

Utilizing the generalized sampling formulation, it was shown in [3] that recurrent nonuniform sampling can be used to lower the sampling density required for perfect reconstruction of 2-D bandlimited signals below the Nyquist rate. Whereas the Nyquist rate corresponds to the highest frequencies in the signal spectral support, the aforementioned sub-Nyquist rate can asymptotically achieve the Landau rate [4] [also known as the minimum-rate sampling (MRS)] corresponding to the Lebesgue measure of the signal spectral support. A similar approach enables rate reduction in the case of bandlimited multiband 1D sequences [5], [6] and continuous signals [7].

While in the aforementioned works the actual sampling patterns depend on the exact knowledge of the spectral support, some other work was related to the concept of universal (spectral blind) sampling. Specifically, only the bandlimit and the size of the packed spectral support is assumed known and one

Manuscript received March 13, 2007; revised October 6, 2007. The associate editor coordinating the review of this manuscript and approving it for publication was Dr. Chong-Meng Samson See.

The authors are with the Department of Electrical Engineering, Technion, Haifa 32000, Israel (e-mail: feuer@ee.technion.ac.il).

Color versions of one or more of the figures in this paper are available online at http://ieeexplore.ieee.org.

Digital Object Identifier 10.1109/TSP.2007.914324 is interested in searching for a sampling pattern that will enable reconstruction regardless of the locations of the subintervals. These search algorithms come with a high price tag concerning their complexity. Besides, a universal pattern is obviously a worst case one, hence suboptimal, when the spectral support is known precisely. This work has also been generalized to 2-D signals [8], [9].

A completely different approach to minimum rate sampling of multiband signals was taken in [10]. There, a perfect reconstruction maximally decimated digital filter-bank formulation was used to show that the class of signals that can be sampled at the minimum rate is much larger than was previously known, and that in some cases, the scheme is also far less complicated. However, the authors of [10] did leave several open questions, one of which is how to choose the sampling pattern to maximize reconstruction robustness.

Yet another approach, taken in [11], shows that signals comprising two disjoint spectral bands (band-pass real signals) can be sampled at their minimum rate. This result was later extended in [12], where signals consisting of a finite number of spectral bands were considered. In both works, however, the authors limit themselves to investigating which sampling patterns cannot be used for reconstruction. These "bad" sampling patterns were also investigated in [13]. While limiting themselves to uniform sampling and to the case of a union of identical subintervals, the authors in [13] investigated the range of sampling rates between the Nyquist and the Landau rates as to which rates enable perfect reconstruction and which do not.

In [14], the authors present reconstruction formulas for recurrent sampling and derive bounds on errors due to mismodeling and additive noise. In [15], they proceed to utilize these bounds as criteria in optimization problems designed to find optimal synthesis filters (interpolation functions) in situations of sub-Nyquist sampling, which are not MRS. They also consider the problem of sampling rate but leave the issue of optimal sampling pattern as an open question.

From a practical viewpoint, sub-Nyquist sampling is very important in several Fourier imaging applications such as sensor array imaging, synthetic aperture radar (SAR), and magnetic resonance imaging (MRI), where the physics of the problem provides us samples of the unknown sparse, in its Fourier domain, object.

This work is closely related to the work presented in [16]-[18]. It deals with finding the most stable sampling pattern for a multiband signal. A detailed discussion of the relationship of our work and that described in the aforementioned papers will be given in the sequel. However, we wish to point out up front that our approach leads to novel results, both analytic and algorithmic with considerable advantages over existing ones. 
Furthermore, while it is not our main goal in this paper, our formulation enabled us to present conditions on the existence of "universal" sampling patterns.

\section{NOTATION AND PROBleM DESCRIPTION}

\section{A. Notation}

Throughout this paper, we use the following notational conventions. Parenthesis are used for continuous time signals [e.g., $s(t)]$ and brackets for discrete time signals (e.g., $s[k]) ; \widehat{f}(\omega)$ denotes the Fourier transform of $f(t)$; vectors are denoted by bold lower case letters and matrices by capital letters; and $A^{H}$ denotes the conjugate transpose of $A . \kappa(A) \triangleq\|A\| \cdot\left\|A^{-1}\right\|$ denotes the condition number $(\mathrm{CN})$ of $A$. When the spectral matrix norm is used, one gets $\kappa(A) \triangleq \sigma_{\max }(A) / \sigma_{\min }(A)$, where $\sigma_{\max }(A)$ and $\sigma_{\min }(A)$ are the maximum and minimum singular values of $A$. Finally, define the spectral support of $s(t)$ as the set $\chi(s)=\{\omega \in \mathbb{R} \mid \widehat{s}(\omega) \neq 0\}$, and define the indicator function of a set $E$ as

$$
1_{E}(x)= \begin{cases}1, & \text { if } x \in E \\ 0, & \text { if } x \notin E .\end{cases}
$$

We will also need some concepts and notation from number theory. For $a, b \in \mathbb{Z}$ and $K \in \mathbb{N}$, we write $a \equiv b(\bmod K)$ if $a-b=l K$ for some $l \in \mathbb{Z}$ and $\operatorname{gcd}(a, b)$ is the largest integer, which divides both $a$ and $b$. A complete residue system $\bmod (K), \operatorname{crs}(K)$, is a set of integers ${\overline{\left\{r_{u}\right\}_{u=1}} K}_{K}$, such that for every $a \in \mathbb{Z}$ there exists a unique $r_{u} \in{\overline{\left\{r_{u}\right.}}_{u=1}^{K}$ for which $a \equiv r_{u}(\bmod K)$. This clearly implies that

$$
r_{q} \equiv r_{u}(\bmod K) \Leftrightarrow u=q, \quad \text { for all } 1 \leq u, q \leq K .
$$

\section{B. Sampling Equations}

In this paper, we focus on $\mathcal{L}^{2}(\mathbb{R})$ signals, which are bandlimited and multiband, meaning that their spectral supports are a finite union of disjoint intervals

$$
\chi(s)=\bigcup_{l=1}^{L}\left(a_{l}, b_{l}\right)
$$

where $a_{1}<b_{1}<a_{2}<b_{2}<\ldots<a_{L}<b_{L}$. For the sake of simplicity of presentation, we assume that the signals are real—all results can readily be extended to complex signals. Hence, we have for every $\omega \in \chi(s)$ also $-\omega \in \chi(s)$ and define the bandwidth and effective bandwidth as

$$
\begin{aligned}
B & =b_{L} \\
B_{\text {eff }} & =\frac{1}{2} \sum_{l=1}^{L}\left(b_{l}-a_{l}\right) .
\end{aligned}
$$

$2 B$ is the rate, for these signals, for which perfect reconstruction is guaranteed from uniform samples (Nyquist rate) and $2 B_{\text {eff }}$ is the minimum average rate, which allows perfect reconstruction from nonuniform samples [4]. Any sampling scheme with average rate in between is referred to as sub-Nyquist sampling. A simple condition for the achievability of MRS is that all band edges are rational multiples of some number. It can be shown, however, [10]-[12] that MRS is achievable under milder conditions.

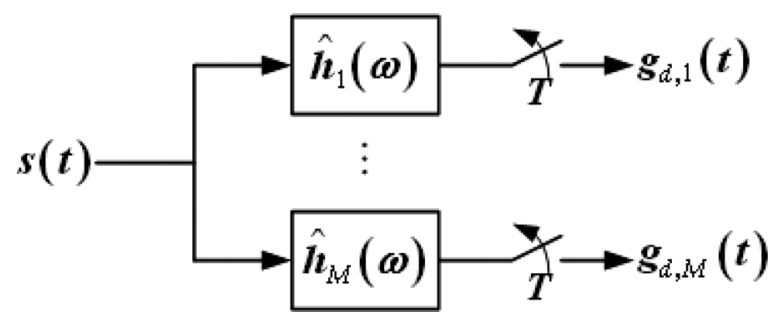

Fig. 1. Analysis part of a generalized sampling scheme.

Because every real number can be approximated by a rational number to any desired accuracy, we can cover $\chi(s)$ by bands that are rational multiples of $B$. Specifically, we can write

$$
\chi(s) \subseteq \bigcup_{k=1}^{K}\left(-B+\frac{2 B n_{k}}{M},-B+\frac{2 B\left(n_{k}+1\right)}{M}\right)
$$

for some $K, M \in \mathbb{N}, K<M$, and the integers $0 \leq n_{1}<n_{2}<$ $\ldots<n_{K} \leq M-1$. We note that by increasing $M$ (which increases $K$ as well) we can make the right-hand side of (4) as close as we want to the left-hand side. We assume in the sequel that a value of $M$ has been chosen and is fixed.

As mentioned earlier, it is well known [1] that by choosing a sampling interval $T_{Q}<\pi / B$, these signals can be perfectly reconstructed from their samples, $\left\{s_{d}[k]=s\left(k T_{Q}\right)\right\}_{k \in \mathbb{Z}}$. However, we will show later (also shown in [7]) that it is also possible to recover such a signal from its periodic nonuniform samples $\left\{s\left(k T+t_{u}\right)\right\}_{k \in \mathbb{Z}, u=0, \ldots, K-1}$, where the average sampling rate $2 \pi K / T$ is lower than the Nyquist rate $2 B$.

Since periodic nonuniform sampling can be formulated as a special case of generalized sampling [19], we use the generalized sampling equation (GSE) formalism. The generalized sampling process is shown in Fig. 1, where $T=M(\pi / B)$ and $g_{d, i}(t)$ are the sampled outputs of the filters. Note that for the sake of simplicity, we define the sampling operation as a modulation (multiplication) by an impulse train, i.e. $g_{d, i}(t) \triangleq$ $g_{i}(t) \cdot \sum_{k \in \mathbb{Z}} \delta(t-k T)$ where $g_{i}(t)$ is the output of the $i$ th filter and $\delta(t)$ is the Dirac delta function. It can readily be seen that we have the relationship

$$
\widehat{g}_{d, i}(\omega)=\frac{1}{T} \sum_{m \in \mathbb{Z}} \widehat{h}_{i}\left(\omega-\frac{2 \pi}{T} m\right) \widehat{s}\left(\omega-\frac{2 \pi}{T} m\right)
$$

or, if we limit ourselves to $\omega \in[B-(2 \pi / T), B)$ and use the fact that $\widehat{s}(\omega)=0$ for all $|\omega|>B$, we have

$$
\begin{aligned}
\widehat{g}_{d, i}(\omega)=\frac{1}{T} \sum_{m=0}^{M-1} \widehat{h}_{i}\left(\omega-\frac{2 \pi}{T} m\right) & \widehat{s}\left(\omega-\frac{2 \pi}{T} m\right), \\
& \text { for } \omega \in\left[B-\frac{2 \pi}{T}, B\right)
\end{aligned}
$$

and in a matrix vector form

$$
H(\omega) \mathbf{s}(\omega)=T \mathbf{g}(\omega)
$$

where (8), shown at the bottom of the next page, holds. Clearly, if $H(\omega)$ is invertible for all $\omega \in[B-(2 \pi / T), B)$, we can calculate $\mathbf{s}(\omega)$ from $\mathbf{g}(\omega)$ and from it $\widehat{s}(\omega)$ for all $\omega \in[-B, B)$. Namely, we can perfectly reconstruct $s(t)$. Interestingly, as was shown in [20], this condition is not only sufficient but also necessary for perfect reconstruction of $s(t)$ bandlimited to $[-B, B)$. 
However, we recall now that in fact $s(t)$ is bandlimited to $\chi(s)$, which satisfies (4). This means that out of the vector $\mathbf{s}(\omega)$ only a subset of $K$ entries will be different from zero and we can reduce accordingly the number of equations also to $K$. Namely, we receive now

$$
H_{K}(\omega) \mathbf{s}_{K}(\omega)=T \mathbf{g}_{K}(\omega)
$$

where (10), shown at the bottom of the page, holds. We refer to this configuration as the reduced generalized sampling equation for multiband signals.

Finally, for recurrent sampling $\left\{s\left(k T+t_{u}\right)\right\}_{k \in \mathbb{Z}, u=0, \ldots, K-1}$, by defining the filters

$$
\widehat{h}_{u}(\omega)=e^{j \omega t_{u}}
$$

we have a special case of the GSE. $H_{K}(\omega)$ becomes

$$
H_{K}(\omega)=\operatorname{diag}\left\{e^{j \omega t_{u}}\right\} \cdot \widetilde{H}_{K}
$$

where

$$
\widetilde{H}_{K}=\left[\begin{array}{ccc}
e^{-j \frac{2 \pi t_{1} n_{1}}{T}} & \cdots & e^{-j \frac{2 \pi t_{1} n_{K}}{T}} \\
\vdots & \ddots & \vdots \\
e^{-j \frac{2 \dot{\pi}_{K} n_{1}}{T}} & \cdots & e^{-j \frac{2 \pi t_{K} n_{K}}{T}}
\end{array}\right] .
$$

As $\operatorname{diag}\left\{e^{j \omega t_{u}}\right\}$ is always nonsingular, the necessary and sufficient condition for perfect reconstruction of the multiband signal $s(t)$ from the data $\left\{s\left(k T+t_{u}\right)\right\}_{k \in \mathbb{Z}, u=0, \ldots, K-1}$ is that the matrix $\widetilde{H}_{K}$ is nonsingular.

Remark 1: For the actual reconstruction formulas (or, equivalently, the synthesis filters), the reader is referred to [21].

\section{Problem Statement}

As discussed previously, the reconstruction of the signal $s(t)$ depends on our ability to solve a set of linear equations. Hence, it is hardly surprising that the stability (or robustness) of the reconstruction process depends, as was shown in [22], on the condition number of the matrix $\widetilde{H}_{K}$. Indeed, we readily observe that for the set of linear equations $\mathbf{y}=A \mathbf{x}$, the relative error of the solution satisfies $\|\Delta \mathbf{x}\| /\|\mathbf{x}\| \leq \kappa(A)\|\Delta \mathbf{y}\| /\|\mathbf{y}\|$. Hence, it would be desirable to make this condition number as small as possible. With this observation, we can formally state our problem.

Problem 1: Given $\mathbf{n}=\left[\begin{array}{llll}n_{1} & n_{2} & \cdots & n_{K}\end{array}\right]^{T} \in \mathbb{Z}^{K}$, where $0 \leq n_{1}<n_{2}<\ldots<n_{K} \leq M-1$ and $\widetilde{H}_{K}$ as defined in $(13)$, find $\mathbf{t}=\left[\begin{array}{llll}t_{1} & t_{2} & \cdots & t_{K}\end{array}\right]^{T} \in[0, T)^{K}$ that minimizes $\kappa\left(\widetilde{H}_{K}\right)$.

Unfortunately, a solution (either a closed-form solution or a polynomial time optimization algorithm) for this problem proved elusive. As a result, in [18], where a related problem was addressed, the authors limit themselves to $t_{u}=(T / M) l_{u}$, $l_{u} \in\{0,1, \ldots, M-1\}$. In that case, the problem can be stated as a combinatorial problem, testing all the possible combinations of $K$ integers out of $\{0,1, \ldots, M-1\}$ and choosing the best combination. However, for increasing values of $M$ and $K$, this approach also becomes quickly unfeasible.

We choose a different route. By constraining $t$ to be of the form

$$
\mathbf{t}=\tau \frac{T}{K}\left[\begin{array}{llll}
0 & 1 & \cdots & K-1
\end{array}\right]^{T}
$$

$$
\begin{aligned}
H(\omega) & =\left[\begin{array}{cccc}
\widehat{h}_{1}(\omega) & \widehat{h}_{1}\left(\omega-\frac{2 \pi}{T}\right) & \ldots & \widehat{h}_{1}\left(\omega-\frac{2 \pi}{T}(M-1)\right) \\
\widehat{h}_{2}(\omega) & \widehat{h}_{2}\left(\omega-\frac{2 \pi}{T}\right) & \ldots & \widehat{h}_{2}\left(\omega-\frac{2 \pi}{T}(M-1)\right) \\
\vdots & \vdots & \ddots & \vdots \\
\widehat{h}_{M}(\omega) & \widehat{h}_{M}\left(\omega-\frac{2 \pi}{T}\right) & \ldots & \widehat{h}_{M}\left(\omega-\frac{2 \pi}{T}(M-1)\right)
\end{array}\right] \\
\mathbf{s}(\omega) & =\left[\begin{array}{lllll}
\widehat{s}(\omega) & \widehat{s}\left(\omega-\frac{2 \pi}{T}\right) & \ldots & \widehat{s}\left(\omega-\frac{2 \pi}{T}(M-1)\right)
\end{array}\right]^{T} \\
\mathbf{g}(\omega) & =\left[\begin{array}{llll}
\widehat{g}_{d, 1}(\omega) & \widehat{g}_{d, 2}(\omega) & \ldots & \widehat{g}_{d, M}(\omega)
\end{array}\right]^{T} .
\end{aligned}
$$

$$
\begin{aligned}
& H_{K}(\omega)= {\left[\begin{array}{cccc}
\widehat{h}_{1}\left(\omega-\frac{2 \pi n_{1}}{T}\right) & \widehat{h}_{1}\left(\omega-\frac{2 \pi n_{2}}{T}\right) & \ldots & \widehat{h}_{1}\left(\omega-\frac{2 \pi n_{K}}{T}\right) \\
\widehat{h}_{2}\left(\omega-\frac{2 \pi n_{1}}{T}\right) & \widehat{h}_{2}\left(\omega-\frac{2 \pi n_{2}}{T}\right) & \ldots & \widehat{h}_{2}\left(\omega-\frac{2 \pi n_{K}}{T}\right) \\
\vdots & \vdots & \ddots & \vdots \\
\widehat{h}_{K}\left(\omega-\frac{2 \pi n_{1}}{T}\right) & \widehat{h}_{K}\left(\omega-\frac{2 \pi n_{2}}{T}\right) & \cdots & \widehat{h}_{K}\left(\omega-\frac{2 \pi n_{K}}{T}\right)
\end{array}\right] } \\
& \mathbf{s}_{K}(\omega)=\left[\begin{array}{llll}
\widehat{s}\left(\omega-\frac{2 \pi n_{1}}{T}\right) & \widehat{s}\left(\omega-\frac{2 \pi n_{2}}{T}\right) & \ldots & \widehat{s}\left(\omega-\frac{2 \pi n_{K}}{T}\right)
\end{array}\right]^{T} \\
& \mathbf{g}_{K}(\omega)=\left[\begin{array}{llll}
\widehat{g}_{d, 1}(\omega) & \widehat{g}_{d, 2}(\omega) & \cdots & \widehat{g}_{d, K}(\omega)
\end{array}\right]^{T}
\end{aligned}
$$


where $\tau \in \mathbb{R}$ is a free parameter to be chosen, $\widetilde{H}_{K}$ becomes a function of $\tau$

$$
\begin{aligned}
& \widetilde{H}_{K}(\tau) \\
& =\left[\begin{array}{cccc}
1 & 1 & \cdots & 1 \\
e^{-j \frac{2 \pi n_{1}}{K} \tau} & e^{-j \frac{2 \pi n_{2}}{K} \tau} & \cdots & e^{-j \frac{2 \pi n_{K}}{K} \tau} \\
\vdots & \vdots & \ddots & \vdots \\
e^{-j \frac{2 \pi(K-1) n_{1}}{K} \tau} & e^{-j \frac{2 \pi(K-1) n_{2}}{K} \tau} & \cdots & e^{-j \frac{2 \pi(K-1) n_{K}}{K} \tau}
\end{array}\right] .
\end{aligned}
$$

We are now ready to restate our problem.

Problem 2: Given $\mathbf{n}=\left[n_{1} n_{2} \cdots n_{K}\right]^{T} \in \mathbb{Z}^{K}$, where $0 \leq n_{1}<n_{2}<\ldots<n_{K} \leq M-1$ and $\widetilde{H}_{K}(\tau)$ as defined in (15), find $\tau \in \mathbb{R}$ that minimizes $J(\tau)=\kappa\left(\widetilde{H}_{K}(\tau)\right)$.

Remark 2: While we have defined $\tau \in \mathbb{R}$, because of (13), we observe that for every resulting $t_{u}=\tau(T / K) u$ in (14), we get the same matrix $\widetilde{H}_{K}$ if we take $t_{u}=(\tau(u / K)-\lfloor\tau(u / K)\rfloor) T$.

\section{Robust ReCURREnt SAMPLING PATterns}

When comparing Problem 1, Problem 2, and the results in [18], we note that while the goals in all three are the same, the search for the optimal sampling pattern is done in different regions. In Problem 1, the search is in $\mathcal{S}_{1}=\left\{\mathbf{t}: 0 \leq t_{u}<T\right\}$; in [18], it is in $\mathcal{S}_{B}=\left\{\mathbf{t}: t_{u}=(T / M) l_{u}, l_{u} \in\{0,1, \ldots, M-\right.$ $1\}\}$; and in Problem 2, the search is in $\mathcal{S}_{2}=\left\{\mathbf{t}: t_{u}=\right.$ $(\tau(u / K)-\lfloor\tau(u / K)\rfloor) T, \tau \in \mathbb{R}\}$ (see Remark 2). Clearly, $\mathcal{S}_{B} \subset \mathcal{S}_{1}$ and $\mathcal{S}_{2} \subset \mathcal{S}_{1}$, hence Problem 1 is the most general. Since the choice $\tau=l K / M, l \in \mathbb{Z}$, results in $\mathbf{t} \in \mathcal{S}_{2}$ and $\mathbf{t} \in \mathcal{S}_{B}$, we conclude that $\mathcal{S}_{B} \cap \mathcal{S}_{2} \neq \varnothing$. On the other hand, one can readily find patterns in $\mathcal{S}_{B}$, which are not contained in the $\mathcal{S}_{2}$ and vice versa, hence, neither region contains the other. This discussion leads to the conclusion that comparisons between the results of these two approaches have limited significance. We wish to point out though, that extensive experiments were conducted and, in the sequel, we do present some experiment results. On the other hand, it is known that $J(\tau)=\kappa\left(\widetilde{H}_{K}(\tau)\right) \geq 1$. An immediate benefit from our choice of search region is that it results in an analytically tractable problem. Specifically, we present necessary and sufficient conditions on $\mathbf{n}$, for which $\kappa\left(\widetilde{H}_{K}\left(\tau_{\text {opt }}\right)\right)=1$ (perfect conditioning) can be achieved and $\tau_{\text {opt }}$ achieves it.

\section{A. Perfect Conditioning}

To state our analytical results, we need the following preliminary results and definitions.

Lemma 1: Let $\widetilde{H}_{K}$ be as in (15). Then, the following holds.

1) Perfect conditioning (i.e., $\kappa\left(\widetilde{H}_{K}(\tau)\right)=1$ ) is achieved if and only if ${\overline{\left\{\tau\left(n_{q}-n_{1}\right)\right\}_{q=1}}}_{q}^{K}$ is a $\operatorname{crs}(K)$.

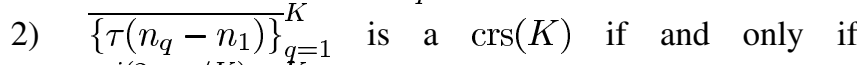
$\left\{e^{-j\left(2 \pi n_{k} / K\right) \tau}\right\}_{k=1}^{K}$ are uniformly spread on the unit circle.

Proof: See Appendix I.

Lemma 2: Let $a, b, c \in \mathbb{Z}$ and $n \in \mathbb{N}$. Then

$$
a c \equiv b c(\bmod n) \Leftrightarrow a \equiv b\left(\bmod \frac{n}{g}\right)
$$

where $g=\operatorname{gcd}(c, n)$

Proof: See [23, Th. 2.1.2].
Theorem 1: Let $\widetilde{H}_{K}(\tau)$ be as in (15) and let $Q=$ $\operatorname{gcd}\left(\left\{n_{q}-n_{1}\right\}_{q=1}^{K}\right)$. Then, there exists a $\tau_{\text {opt }} \in \mathbb{R}$ that achieves perfect conditioning $(\kappa(A)=1)$ if and only if

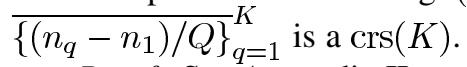

Proof: See Appendix II.

Remark 3: Note that Theorem 1 suggests a simple test for the following question: Given $\mathbf{n}$, is perfect conditioning possible? Calculate $Q=\operatorname{gcd}\left(\left\{n_{q}-n_{1}\right\}_{q=1}^{K}\right)$ and the set $u_{q}=\left\{\left(\left(n_{q}-\right.\right.\right.$ $\left.\left.\left.n_{1}\right) / Q\right)-\left\lfloor\left(n_{q}-n_{1}\right) / K Q\right\rfloor K\right\}_{q=1}^{K}$. Then, perfect conditioning is possible if (and only if) the set $\left\{u_{q}\right\}_{q=1}^{K}$ is a permutation of the set $\{0,1, \ldots, K-1\}$ and it can be achieved with $\tau=1 / Q$ (which is not a unique choice).

While our goal is not to investigate universal sampling patterns, we can completely characterize the universal sampling patterns in the family we chose.

Lemma 3: A sampling pattern of the form (14) is universal if and only if

$$
\frac{\tau m}{K}-\left\lfloor\frac{\tau m}{K}\right\rfloor>0, \quad \text { for all } m=1,2, \ldots, M-1 .
$$

Proof: See Appendix III.

\section{B. Optimal Conditioning}

Suppose now that the given $\mathbf{n}$ is such that perfect conditioning is not possible with the chosen sample patterns. We are still interested in finding the most stable sampling pattern, namely, in solving Problem 2. To do that, we first note some of the properties of the dependence of $\kappa\left(\widetilde{H}_{K}\right)$ on $\tau$. From (15), we have $\widetilde{H}_{K}(\tau+m K)=\widetilde{H}_{K}(\tau)=\left(\widetilde{H}_{K}(-\tau)\right)^{*}$ and, by the definition of $\kappa\left(\widetilde{H}_{K}(\tau)\right)$, we have $\kappa\left(\widetilde{H}_{K}(\tau)\right)=\kappa\left(\left(\widetilde{H}_{K}(\tau)\right)^{*}\right)$. Hence, we can limit our search for optimal $\tau$ to the region $(0,(K / 2)]$.

Remark 4: In fact, we could further restrict the search region by defining $Q=\operatorname{gcd}\left(\left\{n_{q}\right\}_{q=1}^{K}\right)$ and noting that $\widetilde{H}_{K}(\tau+$ $m(K / Q))=\widetilde{H}_{K}(\tau)$. Then, the search region for optimal $\tau$ can be reduced to $(0,(K / 2 Q)]$. However, as $M$ and $K$ increase, the resulting sets $\left\{n_{q}\right\}_{q=1}^{K}$ would generally have $Q=1$. Hence, in the sequel, we prefer to be somewhat more conservative and choose $(0,(K / 2)]$ as our search region.

Let us define the set of angles

$$
\theta_{q}(\tau)=2 \pi\left(\frac{\tau\left(n_{q}-n_{1}\right)}{K}-\left\lfloor\frac{\tau\left(n_{q}-n_{1}\right)}{K}\right\rfloor\right) .
$$

We readily observe that $\theta_{1}(\tau)=0$ and, with $\widetilde{H}_{K}(\tau)$ being Vandermonde, $\kappa\left(\widetilde{H}_{K}(\tau)\right)=\infty$ if and only if $\tau$ is such that $\theta_{q}(\tau)=\theta_{u}(\tau)$ with $q \neq u$. There is a finite number of such $\tau$ in the interval $[0,(K / 2)]$ and they form the set

$$
\begin{aligned}
\mathcal{T}=\left\{\tau=\frac{u K}{\left(n_{i}-n_{j}\right)}:\right. & \leq j<i \leq K, \\
u & \left.=0,1,2, \ldots,\left\lfloor\frac{n_{i}-n_{j}}{2}\right\rfloor\right\} .
\end{aligned}
$$

Let $L+1$ denote the number of distinct members of that set so that $\mathcal{T}=\left\{\tau_{0}=0<\tau_{1}<\ldots<\tau_{L} \leq K / 2\right\}$ and let $\mathcal{I}_{l}=\left(\tau_{l}, \tau_{l+1}\right)$ denote the $L$ intervals between them. We can then rewrite Problem 2 as

$$
\min _{1 \leq l \leq L}\left(\min _{\tau \in \mathcal{I}_{l}} \kappa\left(\widetilde{H}_{K}(\tau)\right)\right)
$$


and denote the optimal $\tau$ as $\tau_{\text {opt }}$. However, finding $\min _{\tau \in \mathcal{I}_{l}} \kappa\left(\widetilde{H}_{K}(\tau)\right)$ directly is still too difficult. Recalling that $\kappa\left(\widetilde{H}_{K}(\tau)\right)=1$ if and only if the angles $\theta_{q}(\tau)$ are uniformly spaced, we replace the criterion $\kappa\left(\widetilde{H}_{K}(\tau)\right)$ with a measure indicating how close to uniformly spaced these angles are. To do that formally, we observe that by the definitions of the set $\mathcal{T}$ and the intervals $\mathcal{I}_{l},\left\lfloor\tau\left(n_{k}-n_{1}\right) / K\right\rfloor$ is constant for all $\tau \in \mathcal{I}_{l}$, hence the order of the angles $\theta_{q}(\tau)$ for $\tau \in \mathcal{I}_{l}$ around the circle does not change. Hence, for each interval $\mathcal{I}_{l}$, there is a permutation matrix $P_{l}$ such that $0=\left(P_{l} \boldsymbol{\theta}(\tau)\right)_{1}<\left(P_{l} \boldsymbol{\theta}(\tau)\right)_{2}<\ldots<\left(P_{l} \boldsymbol{\theta}(\tau)\right)_{K}$, so we can write

$$
\begin{aligned}
\boldsymbol{\theta}(\tau) & =\left[0, \theta_{2}(\tau), \ldots, \theta_{K}(\tau)\right]^{T} \\
& =2 \pi\left(\frac{\tau}{K}\left(\mathbf{n}-n_{1} \mathbf{1}\right)-\mathbf{m}_{l}\right)
\end{aligned}
$$

where 1 is a vector of ones and $\mathbf{m}_{l}=\left[0,\left\lfloor\tau\left(n_{2}-\right.\right.\right.$ $\left.\left.\left.n_{1}\right) / K\right\rfloor, \ldots,\left\lfloor\tau\left(n_{K}-n_{1}\right) / K\right\rfloor\right\rfloor$ (independent of $\tau$ ). The angles are uniformly spaced if for some $\tau \in \mathcal{I}_{l}$

$$
P_{l} \boldsymbol{\theta}(\tau)=\frac{2 \pi}{K} \mathbf{e}_{K}
$$

where

$$
\mathbf{e}_{K}=\left[\begin{array}{c}
0 \\
1 \\
\vdots \\
K-1
\end{array}\right]
$$

Hence, we replace the problem $\tau_{o p t, l}=\arg \min _{\tau \in \mathcal{I}_{l}} \kappa\left(\widetilde{H}_{K}(\tau)\right)$ with

$$
\tilde{\tau}_{o p t, l}=\arg \min _{\tau \in \mathcal{I}_{l}}\left\|P_{l} \boldsymbol{\theta}(\tau)-\frac{2 \pi}{K} \mathbf{e}_{K}\right\|_{p} .
$$

Extensive experiments that we conducted, a sample of which you can see in Fig. 2 , indicated that for $p=2$, we get $\widetilde{\tau}_{\text {opt }, l} \approx$ $\tau_{\text {opt }, l}$, hence that is the norm we chose (note, however, that it is not analytically confirmed). For this choice, (24) becomes a standard least squares problem and we can derive the closedform solution

$$
\widetilde{\tau}_{o p t, l}=\frac{\left(\mathbf{n}-n_{1} \mathbf{1}\right)^{T}\left(K \mathbf{m}_{l}+P_{l}^{T} \mathbf{e}_{K}\right)}{\left(\mathbf{n}-n_{1} \mathbf{1}\right)^{T}\left(\mathbf{n}-n_{1} \mathbf{1}\right)}
$$

For a given $\mathbf{n}$, we propose the following algorithm to find the most stable sampling pattern.

1) Using (19), calculate the set $\mathcal{T}$ and the corresponding intervals $\left\{\mathcal{I}_{l}\right\}_{l=0}^{L-1}$.

2) Calculate a set of interval representing values $\left\{\widetilde{\tau}_{l}\right\}_{l=0}^{L-1}$ as

$$
\tilde{\tau}_{l}=\frac{\tau_{l}+\tau_{l+1}}{2}
$$

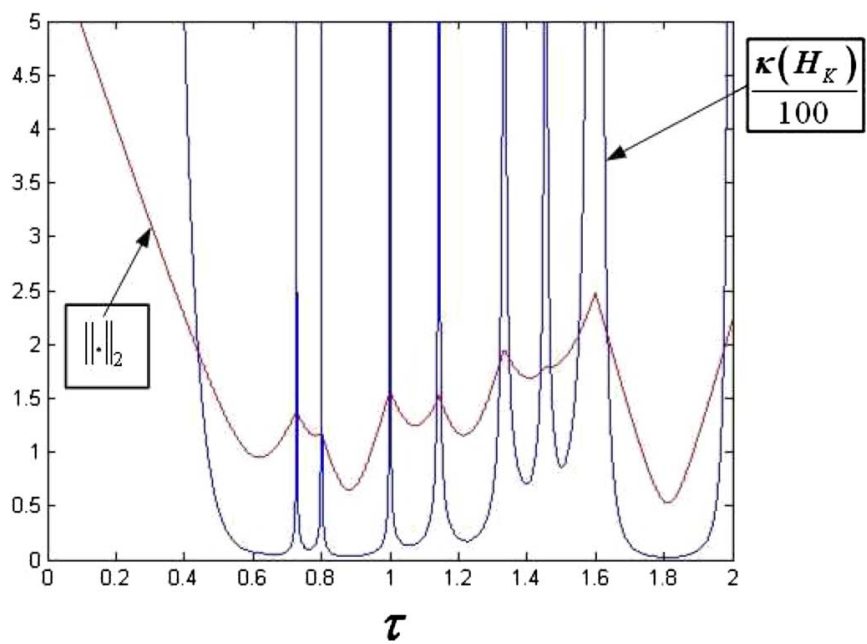

Fig. 2. Example of the correlation between the condition number and the norm-2 measure of uniformity.

3) Calculate $\mathbf{m}_{l}=\left[0,\left\lfloor\widetilde{\tau}_{l}\left(n_{2}-n_{1}\right) / K\right\rfloor, \ldots,\left\lfloor\widetilde{\tau}_{l}\left(n_{K}-n_{1}\right) / K\right\rfloor\right]$ and, using (21), calculate $\boldsymbol{\theta}\left(\widetilde{\tau}_{l}\right)$.

4) Using $\boldsymbol{\theta}\left(\widetilde{\tau}_{l}\right)$, determine the permutation matrix $P_{l}$.

5) Using (25), calculate $\left\{\widetilde{\tau}_{\text {opt }, l}\right\}$ and find $\widetilde{\tau}_{\text {opt }}$, which gives

$$
\underset{\tilde{\tau}_{o p t, l}}{\min } \kappa\left(\widetilde{H}_{K}\left(\widetilde{\tau}_{o p t, l}\right)\right) \text {. }
$$

6) The stable (recurrent) sampling pattern is then given by

$$
\bigcup_{k \in \mathbb{Z}} \bigcup_{u=1}^{K} \frac{\pi M}{B}\left(k+\widetilde{\tau}_{\text {opt }} \frac{u-1}{K}\right) .
$$

1) Computational Issues: Scanning the proposed algorithm, we note that the only step of the algorithm that involves significant computations is Step 5), which requires $L$ times the calculation of the condition number of a $K$-dimensional matrix. An efficient algorithm to calculate the condition number requires $(8 / 3) K^{3}$ (see [24]) operations. Hence, we can estimate the computation load required by the algorithm to be of the order of $(8 / 3) L K^{3}$. The number $L$ depends heavily on the vector $\mathbf{n}$, so we next present an upper bound on its value.

Lemma 4: Let $L+1$ be the number of elements in the set $\mathcal{T}$ as defined in (19) for a given $\mathbf{n}$. Then

$$
L \leq \begin{cases}\frac{1}{4}\left[\left(n_{K}-n_{1}\right)^{2}-1\right], & \text { for odd }\left(n_{K}-n_{1}\right) \\ \frac{1}{4}\left(n_{K}-n_{1}\right)^{2}, & \text { for even }\left(n_{K}-n_{1}\right) .\end{cases}
$$

Proof: It follows directly from (19), when we consider all possible differences $\left(n_{i}-n_{j}\right)$ and ignore possible repetitions of the values $\tau=u K /\left(n_{i}-n_{j}\right)$.

As pointed out earlier, while related work in the literature conducts its search in a different family of sampling patterns, we would like to make some comparisons as far as computational complexity. As the approach is combinatorial-choosing 

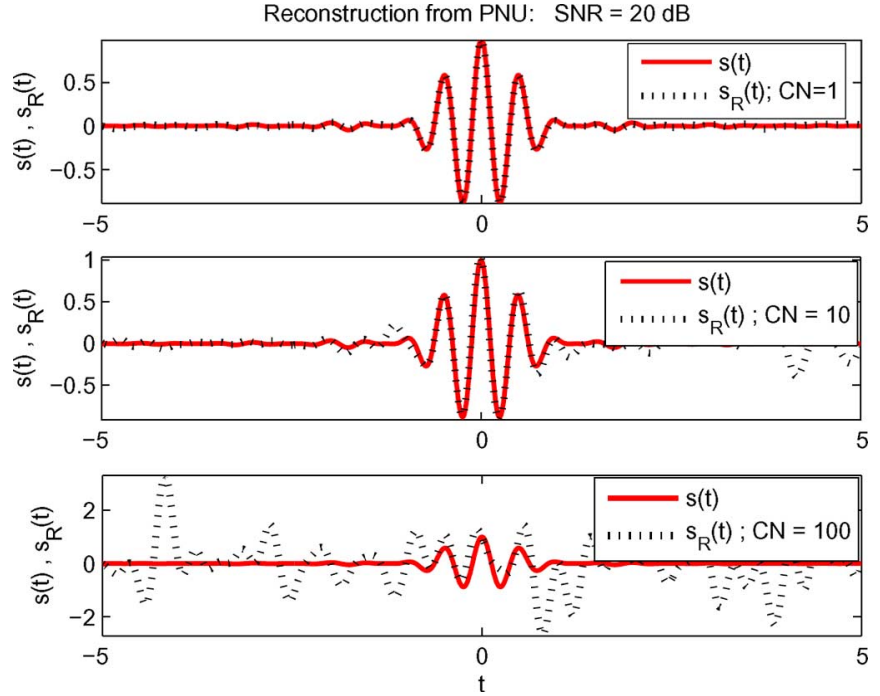

Fig. 3. Condition number effect on the quality of reconstructed signal.

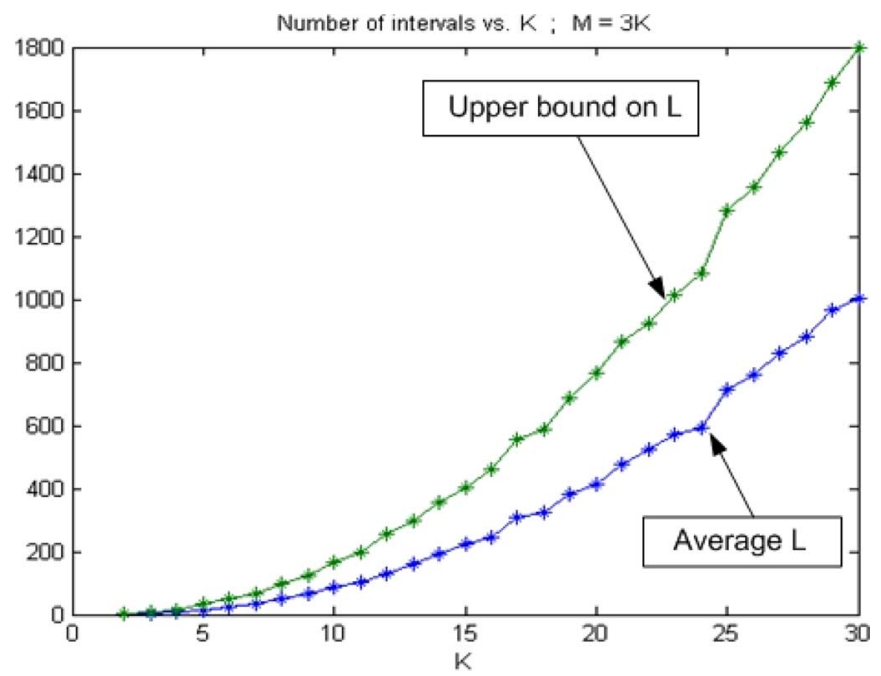

Fig. 4. Tightness on computation upper bound.

the best $K$ out of $M$ rows-it will require calculating the condition number $\left(\begin{array}{c}M \\ K\end{array}\right)$ times. This is clearly, significantly larger than any value we get for the bound on $L$. For instance, if $M=60$ and $K=30$, we get for any choice of $\mathbf{n}, L \leq 870$, while $\left(\begin{array}{l}60 \\ 30\end{array}\right) \geq 10^{17}$. We should point out though, that the literature contains a number of search algorithms that circumvent the brute force approach and ease the computational load but provide only "almost" optimal sampling patterns.

\section{Simulation Results}

We have carried out extensive experiments on the computer to test and verify the claims we make here. A sample of the results is presented in the sequel.

We start by demonstrating the effect of the condition number when reconstructing a signal contaminated by noise, from its sampled values. We remind the reader that the condition number is determined by the choice of the sampling pattern. In Fig. 3,
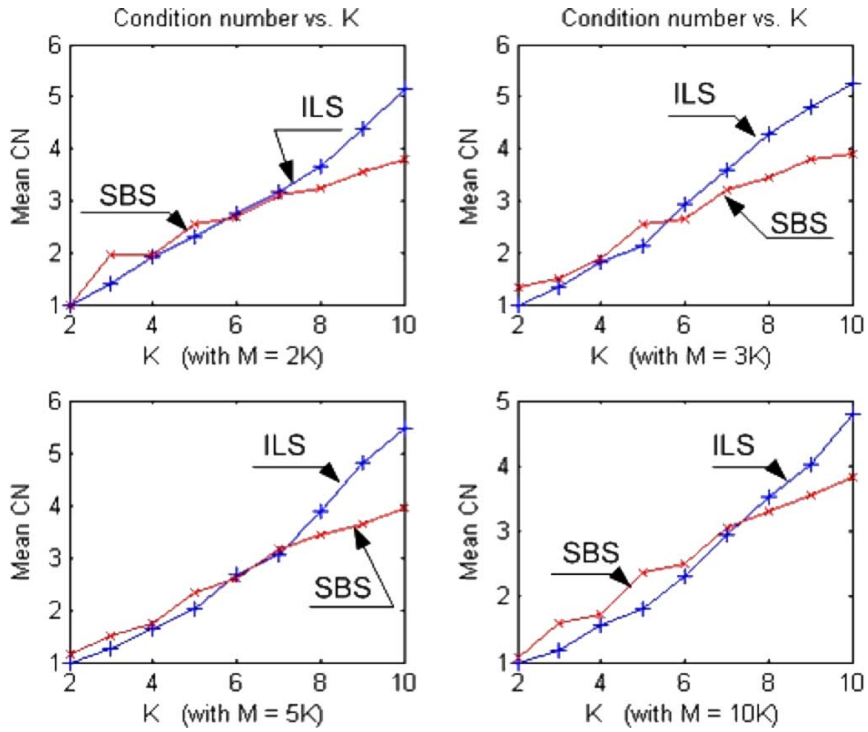

Fig. 5. Performance comparison between the SBS and ILS.

where $s(t)$ and $s_{R}(t)$ are the signal and its reconstructed version, respectively, we clearly see the deterioration of the reconstructed signal quality as the condition number grows. We wish to point out that this experiment has been done with $K=2$; in experiments with a larger $K$, this phenomenon dramatically increase. To see how tight is the upper bound we gave in (28), we chose $M=3 K$ to generate the relationship between $L$ and $K$ by averaging over 25 runs per $K$ with randomly chosen $\mathbf{n}$. The results are shown in Fig. 4. As we stated earlier, there are not many results that deal with the question of sampling pattern selection and most are concerned with finding universal patterns. The few that discuss finding a sampling pattern for a specific spectral support as we did here, use sequential selection algorithms where they choose $K$ rows out of $M$ as described earlier. One of these is described in [16] and referred to as sequential backward selection (SBS). Recall that the SBS searches within $\mathcal{S}_{B}$ as defined earlier. In Fig. 5, we compare the results of using the SBS to our algorithm [referred to as iterative least squares (ILS)] where the condition numbers were averaged over 100 random $\mathbf{n}$ per $K$. We note that for small values of $K$ (and $M$ ) the two algorithms result in sampling patterns with similar condition numbers. As the value of $K$ increases, the SBS algorithm results in more robust patterns. However, this comes at a heavy computational cost as demonstrated in Fig. 6. Finally, as both the ILS (our algorithm) and the SBS are suboptimal, in order to see how close the results are of either to their respective optimal values or to the original problem solution, we chose two simple cases. We considered two examples, $M=15, K=3$ and $M=25, K=3$. In each example, we considered all possible vectors $\mathbf{n}$. As $K$ is sufficiently small, we managed, for each $\mathbf{n}$, to find the optimal sampling patterns in $\mathcal{S}_{1}, \mathcal{S}_{2}$, and $\mathcal{S}_{B}$. In addition, for each $\mathbf{n}$, we have applied the ILS and the SBS. For each $\mathbf{n}$ and resulting sampling patterns, we calculated the corresponding condition numbers. In Fig. 7, we denote by $C N_{1}$, $C N_{2}$, and $C N_{4}$ the condition numbers that corresponds to the search in $\mathcal{S}_{1}$ (the solution of Problem 1), $\mathcal{S}_{2}$ (the solution of 

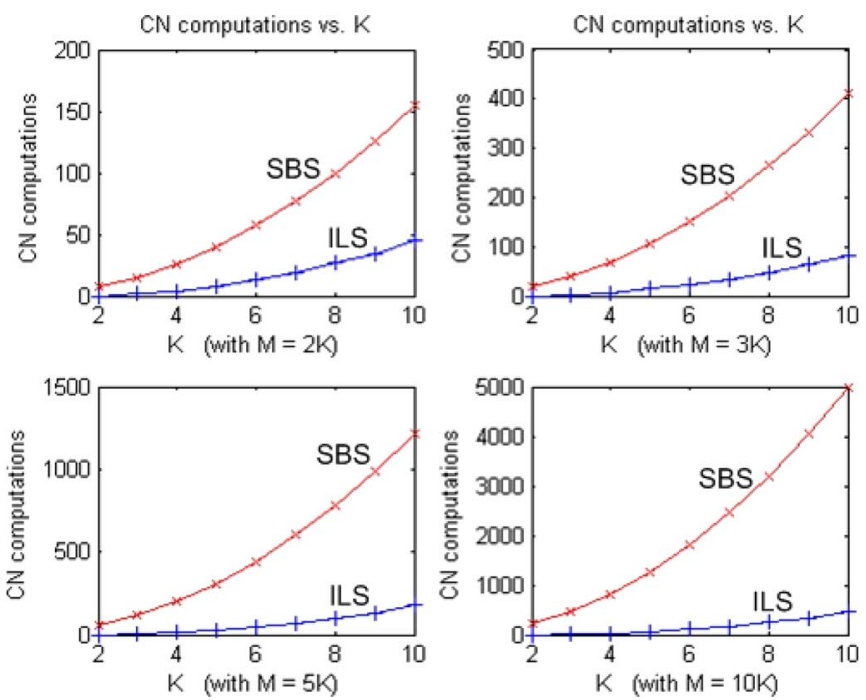

Fig. 6. Computational complexity comparison between the SBS and ILS.

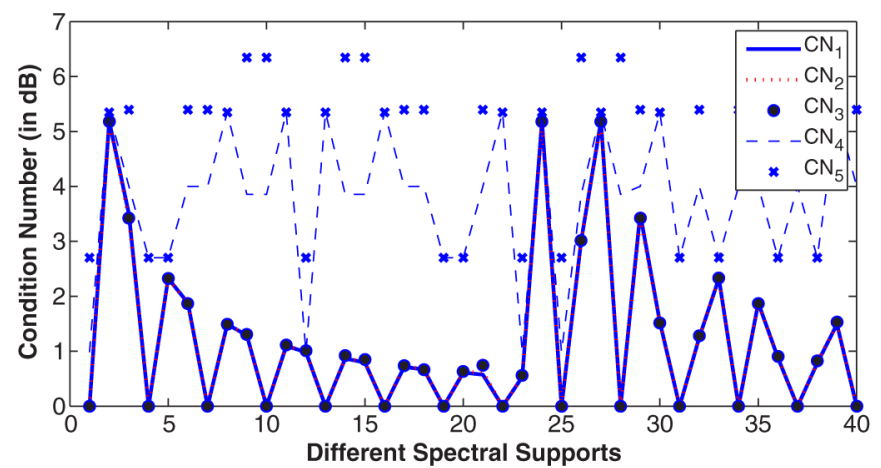

Fig. 7. Comparison of condition numbers for the example $M=25, K=3$.

Problem 2), and $\mathcal{S}_{B}$, respectively. $C N_{3}$ and $C N_{5}$ correspond to the results of using the ILS and the SBS, respectively. We observe first that $C N_{1}$ is less than or equal to all others as expected. Furthermore, whenever perfect conditioning is possible (i.e., $C N_{1}=1$ ), it is possible to achieve it with a pattern of the form in (14) $\left(C N_{2}=1\right)$ and the ILS finds it $\left(C N_{3}=1\right)$. This is not true for the patterns is $S_{B}\left(C N_{4}>1\right)$. Because of space constraints, we did not show here all the results for this example or any of the other example we tried, but all the results maintain the same relationships as previously mentioned.

\section{CONCLUSION}

In this paper, we look at multiband, bandlimited signals and search for robust sub-Nyquist sampling patterns. While previous work was based on a combinatorial, computationally costly search, we introduce a new approach. Instead of a finite set $\mathcal{S}_{B}$, consisting of subsets of $K$ points out of a possible $M$, the sampling patterns considered here are continuously labeled and structurally constrained, the set $\mathcal{S}_{2}$, so that the resulting problem possesses a Vandermonde structure. This approach leads to analytical results-conditions on the spectral support for achievability of perfect conditioning as well as conditions for the existence of universal sampling patterns. For the case when perfect conditioning is not possible, a new algorithm was presented to search for the most robust sampling pattern.

\section{APPENDIX I}

\section{PROOF OF LEMMA 1}

First, we note that

$$
\kappa(A)=1 \Leftrightarrow A^{H} A=c I, \quad \text { for some } c \in \mathbb{R}
$$

which implies that

$$
\begin{aligned}
\kappa\left(\widetilde{H}_{K}\right)=1 \Leftrightarrow\left(\left(\widetilde{H}_{K}\right)^{H} \widetilde{H}_{K}\right)_{u, q} & =0, \\
& \text { for all } 1 \leq u \neq q \leq K .
\end{aligned}
$$

As from (15), we have that

$$
\begin{aligned}
& \left(\left(\widetilde{H}_{K}\right)^{H} \widetilde{H}_{K}\right)_{u, q} \\
& =\sum_{l=0}^{K-1} e^{\frac{j 2 \pi l \tau\left(n_{u}-n_{q}\right)}{K}} \\
& = \begin{cases}K, & \text { if } \tau\left(n_{u}-n_{q}\right) \equiv 0(\bmod K) \\
\frac{1-e^{j 2 \pi \tau\left(n_{u}-n_{q}\right)}}{1-e^{\frac{j \pi \tau\left(n_{u}-n_{q}\right)}{K}}}, & \text { otherwise }\end{cases}
\end{aligned}
$$

when combined with (30) we get for all $1 \leq u \neq q \leq K$

$$
\begin{aligned}
\kappa\left(\widetilde{H}_{K}\right)=1 \Leftrightarrow & \frac{1-e^{j 2 \pi \tau\left(n_{u}-n_{q}\right)}}{1-e^{\frac{j 2 \pi\left(n_{u}-n_{q}\right)}{K}}}=0 \\
\Leftrightarrow & \left\{\tau\left(n_{u}-n_{q}\right) \not \equiv 0(\bmod K)\right\} \\
& \wedge\left\{\tau\left(n_{u}-n_{q}\right) \cdot \in \mathbb{Z}\right\}
\end{aligned}
$$

Hence

$$
\kappa\left(\widetilde{H}_{K}\right)=1 \Leftrightarrow{\overline{\left\{\tau\left(n_{u}-n_{1}\right)\right\}_{u=1}^{K}}}_{u \text { is a } \operatorname{crs}(K)}
$$

which establishes 1).

Then, 2) follows directly from the definitions of a complete residue system and the angles.

\section{APPENDIX II \\ PROOF OF THEOREM 1}

From Lemma 1, we have that $\kappa\left(\widetilde{H}_{K}\right)=1 \Leftrightarrow$; there exists a $\tau$ such that $\overline{\left\{\tau\left(n_{q}-n_{1}\right)\right\}_{q=1}^{K}}$ is a $\operatorname{crs}(K)$, so all we need to show is that there exists a $\tau \in \mathbb{R}$ such that $\overline{\left\{\tau\left(n_{q}-n_{1}\right)\right\}_{q=1}^{K}}$ is $\operatorname{a~} \operatorname{crs}(K) \Leftrightarrow{\overline{\left\{\left(n_{q}-n_{1}\right) / Q\right\}}}_{q=1}^{K}$ is a $\operatorname{crs}(K)$.

For sufficiency, let ${\overline{\left\{\left(n_{q}-n_{1}\right) / Q\right\}_{q=1}}}_{q}^{K}$ be a $\operatorname{crs}(K)$. Let us choose $\tau=1 / Q$. Then, $\tau\left(n_{q}-n_{1}\right)=\left(n_{q}-n_{1}\right) / Q$ and we are done.

For necessity, assume that there exists $\tau \in \mathbb{R}$ such that $\overline{\left\{\tau\left(n_{q}-n_{1}\right)\right\}_{q=1}^{K}}$ is a $\operatorname{crs}(K)$. Then, we have

$$
\begin{aligned}
\tau\left(n_{q}-n_{1}\right) & =\tau Q \frac{n_{q}-n_{1}}{Q} \in \mathbb{Z} \\
& \Rightarrow \tau Q=R \in \mathbb{Z} \\
& \Rightarrow \tau=\frac{R}{Q} .
\end{aligned}
$$

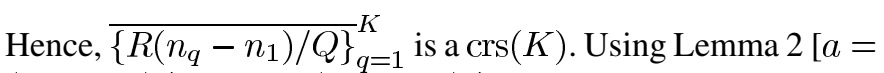
$\left(n_{q}-n_{1}\right) / Q, b=\left(n_{u}-n_{1}\right) / Q, c=R$, and $\left.n=K\right]$, 
we have $R\left(\left(n_{q}-n_{1}\right) / Q\right) \equiv\left(R\left(\left(n_{u}-n_{1}\right) / Q\right)\right)(\bmod K)$ if and only if $\left(n_{q}-n_{1}\right) / Q \equiv\left(n_{u}-n_{1}\right) / Q(\bmod (K / g))$ where $g=\operatorname{gcd}(R, K)$. However, as a $\operatorname{crs}(K / g)$ contains exactly $K / g$ distinct integers, if $K / g<K$, the set $\left\{\left(n_{q}-n_{1}\right) / Q\right\}_{q=1}^{K}$ must contain at least two integers $1 \leq u \neq q \leq K$ such that $\left(n_{q}-n_{1}\right) / Q \equiv\left(n_{u}-n_{1}\right) / Q(\bmod (K / g))$. By the above, this implies that $R\left(\left(n_{q}-n_{1}\right) / Q\right) \equiv\left(R\left(n_{u}-n_{1}\right) / Q\right)(\bmod$ $K)$, which contradicts the fact that $\overline{\left\{R\left(n_{q}-n_{1}\right) / Q\right\}_{q=1}^{K}}$ is a $\operatorname{crs}(K)$. Hence, necessarily, $g=1$ so that ${\overline{\left\{\left(n_{q}-n_{1}\right) / Q\right\}_{q=1}}}^{K}$ is a $\operatorname{crs}(K)$, which completes the proof.

\section{APPENDIX III PROOF OF LEMMA 3}

Since the matrix $\widetilde{H}_{K}(\tau)$ is Vandermonde with the generating row given by $\left\{e^{-j\left(2 \pi \tau n_{q} / K\right)}\right\}_{q=1}^{K}$, it will be singular if and only if

$$
e^{-j \frac{2 \pi \tau n_{u}}{K}}=e^{-j \frac{2 \pi \tau n_{q}}{K}}
$$

for some $u<q$. Namely, if and only if

$$
\frac{\tau\left(n_{q}-n_{u}\right)}{K} \in \mathbb{Z}
$$

and as, by definition, $1 \leq n_{q}-n_{u} \leq M-1$ for $u<q$, (33) is equivalent to

$$
\frac{\tau\left(n_{q}-n_{u}\right)}{K}-\left\lfloor\frac{\tau\left(n_{q}-n_{u}\right)}{K}\right\rfloor=0 .
$$

The proof is complete.

\section{REFERENCES}

[1] C. E. Shannon, "Communications in the presence of noise," Proc. IRE, vol. 37, pp. 10-21, Oct. 1949.

[2] A. J. Jerri, "The Shannon sampling theorem-Its various extensions and applications: A tutorial review," Proc. IEEE, vol. 65, no. 11, pp. 1565-1596, Nov. 1977.

[3] K. F. Cheung and M. I. R. J, "Imaging sampling below the Nyquist density without aliasing," J. Opt. Soc. Amer. A, Opt. Image Sci., vol. 7, pp. 92-105, Jan. 1990.

[4] H. J. Landau, "Necessary density conditions for sampling and interpolation of certain entire functions," ACTA Mathematica, vol. 117, pp. 37-52, 1967.

[5] P. Vaidyanathan, "Multirate digital filters, filter banks, polyphase networks, and applications: A tutorial," Proc. IEEE, vol. 78, no. 1, pp. 56-93, Jan. 1990 .

[6] B. Foster and C. Herley, "Exact reconstruction from periodic nonuniform samples," in Proc. IEEE Int. Conf. Acoust. Speech Signal Process., 1995, vol. 2, pp. 1452-1455.

[7] P. Feng and Y. Bresler, "Spectrum-blind minimum-rate sampling and reconstruction of multiband signals," in Proc. IEEE Int. Conf. Acoust. Speech Signal Process., 1996, vol. 3, pp. 1688-1691.

[8] Y. Bresler and P. Feng, "Spectrum-blind minimum rate sampling and reconstruction of 2-d multiband signals," in Proc. IEEE Int. Conf. Acoust. Speech Signal Process., 1996, pp. 701-704.

[9] R. Venkataramani and Y. Bresler, "Further results om spectrum blind sampling of 2-d signals," in Proc. IEEE Int. Conf. Acoust. Speech Signal Process., 1998, pp. 752-756.

[10] C. Herley and P. W. Wong, "Minimum rate sampling and reconstruction of signals with arbitrary frequency support," IEEE Trans. Inf. Theory, vol. 45, no. 5, pp. 1555-1564, Jul. 1999.

[11] A. Kohlenberg, "Exact interpolation of band-limited functions," $J$. Appl. Phys., vol. 24, no. 12, pp. 1432-1436, Dec. 1953.

[12] Y. P. Lin and P. P. Vaidyanathan, "Periodically nonuniform sampling of band-path signals," IEEE Trans. Circuits Systems. II, Analog Digit. Signal Process., vol. 45, no. 3, pp. 340-351, Mar. 1998.

[13] M. G. Beaty and M. M. Dodson, "The distribution of sampling rates for signals with equally wide, equally spaced spectral bands," SIAM J. Appl. Math., vol. 53, no. 3, pp. 893-906, June 1993.

[14] R. Venkataramani and Y. Bresler, "Perfect reconstruction formulas and bounds on aliasing error in sub-Nyquist nonuniform sampling of multiband signals," IEEE Trans. Inf. Theory, vol. 46, no. 6, pp. 2173-2183, Sep. 2000.

[15] R. Venkataramani and Y. Bresler, "Optimal sub-Nyquist nonuniform sampling and reconstruction for multiband signals," IEEE Trans. Signal Process., vol. 49, no. 10, pp. 2301-2313, Oct. 2001.

[16] S. J. Reeves and L. P. Heck, "Selection of observations in signal reconstruction," IEEE Trans. Signal Process., vol. 43, no. 3, pp. 788-791, Mar. 1995.

[17] Y. Gao and S. J. Reeves, "Optimal k-space sampling in MRSI for images with a limited region of support," IEEE Trans. Med. Imag., vol. 19 , no. 12, pp. 1168-1178, Dec. 2000.

[18] N. D. Blakeley, P. J. Bones, R. P. Millane, and P. Renaud, "Efficient frequency-domain sample selection for recovering limited-support images," J. Opt. Soc. Amer. A., Opt. Image Sci., vol. 20, pp. 67-77, Jan. 2003.

[19] A. Papoulis, "Generalized sampling expansion," IEEE Trans. Circuits Syst., vol. CS-24, no. 11, pp. 652-654, Nov. 1977.

[20] A. Feuer, "On the necessity of Papoulis result for multidimensional generalized sampling expansion (GSE)," IEEE Signal Process. Lett., vol. 11, no. 4, pp. 420-422, Apr. 2004.

[21] A. Feuer and G. C. Goodwin, "Reconstruction of multidimensional bandlimited signals from nonuniform and generalized samples," IEEE Trans. Signal Process., vol. 53, no. 11, pp. 4273-4282, Nov. 2005.

[22] M. Unser and J. Zerubia, "Generalized sampling: Stability and performance analysis," IEEE Trans. Signal Process., vol. 45, no. 12, pp. 2914-2950, Dec. 1997.

[23] R. A. Mollin, Fundamental Number Theory With Applications, ser. Discrete Mathematics and Its Applications, K. H. Rosen, Ed. Boca Raton, FL: CRC Press, 1998.

[24] M. Gu, J. W. Demmel, and I. Dhillon, "Efficient computation of the singular value decomposition with applications to least squares problems,” Knoxville, TN, Tech. Rep. CS-94-257, 1994.

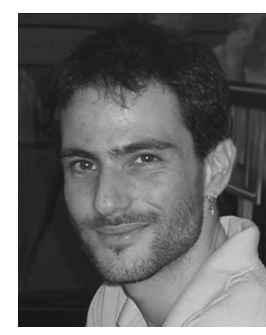

ship.
Lihu Berman received the B.Sc. and M.Sc. degrees from the Department of Electrical Engineering, The Technion-Israel Institute of Technology, Haifa, Israel, in 2003 and 2006, respectively, where he is currently working towards the Ph.D. degree. While doing research for the M.Sc. degree, he worked closely with Prof. A. Feuer.

From 1997 to 2000, he served in the Israeli Military Intelligence.

Mr. Berman is a graduate of the Technion's excellence program and a recipient of the Gutwirth fellow-

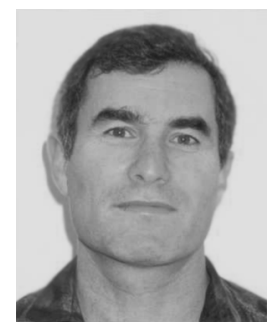

Arie Feuer (S'74-M'76-SM'93-F'04) received the B.Sc. and M.Sc. in mechanical engineering from the Technion-Israel Institute of Technology, Haifa, Israel, in 1967 and 1973, respectively, and the Ph.D. degree from Yale University, New Haven, CT, in 1978.

From 1967 to 1970, he was with Technomatics Inc. working on the design of automatic machines. From 1978 to 1983, he worked for Bell Labs in network performance evaluation. In 1983, he joined the Faculty of Electrical Engineering at the Technion were he is currently a Professor and Head of the Control and Robotics Laboratory. His current research interests include resolution enhancement of digital images and videos; sampling and combined representations of signals and images; and adaptive systems in signal processing and control 